\title{
Sonsuz Grupların Çapraz Çarpımı için Tam Yeniden Yazma Sistemi
}

\author{
Ayşe Çevik ${ }^{1}$, Eylem Güzel Karpuz ${ }^{*} \mathbb{D}^{\mathbb{D}}$, Esra Kırmızı Çetinalp ${ }^{1}$ \\ ${ }^{1}$ Karamanoğlu Mehmetbey Üniversitesi, Kamil Özdağ Fen Fakültesi, Matematik Bölümü \\ Karaman/Türkiye
}

Geliş / Received: 25/10/2019, Kabul / Accepted: 21/02/2020

\begin{abstract}
Öz
$\mathrm{Bu}$ çalışmada; ilk olarak iki serbest grubun çapraz çarpımının monoid sunuşu için kelime probleminin çözülebilir olduğu incelenmiştir. Bunun için teorik bilgisayar biliminde de önemli bir yeri olan yeniden yazma sistemi tekniği kullanılmıştır. Daha sonra ise rankları 2 olan iki serbest abelyan grubun çapraz çarpımının sunuşu elde edilmiştir.

Anahtar Kelimeler: Yeniden Yazma Sistemi, Kelime Problemi, Çapraz Çarpum.
\end{abstract}

\section{Complete Rewriting System for Crossed Product of Infinite Groups}

\begin{abstract}
In this work, firstly, it is investigated the solvability of the word problem for monoid presentation of crossed product of two free groups. To do that it is used rewriting system method which has an important role in theoretical computer science. Then it is obtained a presentation for crossed product of two free abelian groups of rank 2.
\end{abstract}

Keywords: Rewriting System, Word Problem, Crossed Product.

\section{Giriş}

Kombinatoryal Grup Teoride oldukça önemli bir çalışma alanı oluşturan karar verme problemlerinin, geometrik grup teorisi ve son y1llarda da teorik bilgisayar bilimi ile olan ilişkileri önem kazanan konular arasındadır. $\mathrm{Bu}$ çalışmanın temel konularından biri olan kelime problemi, 1911 yılında Max Dehn tarafindan ortaya atılan üç ana karar verme problemlerinden biridir (Dehn, 1911). Bu problemin hangi grup/grup çarpımları (monoid, yarı grup sınıfları) için çözülebilir, hangileri için çözülemez olduğu yönündeki çalışmalar oldukça önemlidir (Miller, 1992).
Grup genişlemesinin temelini oluşturan direkt ve yarı-direkt çarpımın genellemesi olan çapraz çarpım; devirli gruplarda (Agore, Militaru, 2008; Agore, Fratila, 2010) , Lie gruplarında (Rudkovskii, 1997), Hopf cebirinde (Carbani ve ark., 2010) ve $C^{*}$ cebirlerinde (Grundling ve Neeb, 2014), halkalarda (Abuhlail, 2005), topolojik uzayda, vb... alanlarda çalışılmıştır. Çapraz çarpım yapısının bu kadar önemli olması, bu çarpım ile herhangi çarpımın birleştirilmesi sonucu oluşturulan yeni cebirsel yapının özellikleri nedir ya da bu çarpımı kapsayan daha geniş cebirsel yapılar oluşturulabilir mi? sorularını gündeme getirmektedir. $\mathrm{Bu}$ sorulara grup teori alanında verilen cevaplar

*Sorumlu Yazar: eylem.guzel@kmu.edu.tr 
olarak (Emin vd., 2013; Çetinalp, 2016; Çetinalp vd., 2016; Çetinalp ve Karpuz, 2018; Karpuz ve Çetinalp, 2018) çalışmalarına bakılabilir. Grup teorisinde yapılan bu çalışmalardan hareketle, bu çalışmada ilk olarak rankları (üreteç elemanların sayısı) 1 olan iki serbest grubun çapraz çarpımının monoid sunuşu için tam yeniden yazma sistemi bulunacaktır. Daha sonra ise, rankları 2 olan iki serbest abelyan grubun çapraz çarpımının sunuşu elde edilecektir. Böylelikle grup teoride çapraz çarpım gibi önemli bir genişleme türü üzerine katkı sağlanacaktır.

\section{Temel Tanım ve Teoremler}

$\mathrm{Bu}$ bölümde bir grubun kelime probleminin çözülebilirliği, (pozitif) yeniden yazma sistemi ve grupların çapraz çarpımı ile ilgili bazı tanım ve teoremlere yer verilmiştir.

\subsection{Kelime problemi}

Elemanlar ve gruplar hakkında çeşitli soruları cevaplayan bir algoritmanın (veya metodun) varlığı problemine karar verme problemleri denir. Eğer verilen bir problemi çözmek için bir algoritma (veya metot) varsa bu karar verme problemine çözülebilir, böyle bir algoritma yoksa o zaman da bu karar verme problemine çözülemez denir. En önemli karar verme problemleri kelime, eşlenik ve izomorfizma problemleridir. $\mathrm{Bu}$ problemler ve türevleri ile ilgili detaylı bilgilere (Miller, 1992) den ulaş1labilir.

Kelime Problemi: $G$ sonlu sunuşlu bir grup olmak üzere, bu grup için kelime problemi $G$ nin üreteçleri ile oluşturulan keyfi bir $w$ kelimesinin bu grubun birimine eşit olup olmadığına karar veren bir algoritmanın varlığının araştırılması problemidir.

\subsection{Yeniden yazma sistemi}

$\mathrm{Bu}$ alt bölümde verilen bilgilere (Book, 1987), (Book ve Otto, 1993) ve (Sims, 1994) kaynaklarından ulaşılabilir.

Sonlu bir $X$ alfabesi için, $X^{*}$ bu alfabedeki harflerden oluşan bütün kelimelerin kümesi ve $\lambda$ boş kelime olsun. $X$ üzerindeki yeniden yazma kuralı $(l, r) \in X^{*} \times X^{*}$ şeklindeki sıralı çiftlerdir. Bu kural $l \rightarrow r$ şeklinde gösterilir. Buradaki $l$ kelimesi sol yan, $r$ kelimesi ise sağ yan diye adlandirılır. Yeniden yazma sistemi $X$ üzerindeki yeniden yazma kurallarının bir kümesidir ve bu sistem $R$ ile gösterilir. $X^{*}$ kümesindeki kelimeler arasındaki bu bağıntı $(\rightarrow r)$ için aşağıdaki kural tanımlanır:

$X$ üzerindeki $u$ ve $v$ pozitif kelimeler olmak üzere, $u \rightarrow_{R} v$ olması için gerek ve yeter koşul $x, y \in X^{*}$ ve $(l, r) \in R \quad$ için $u=x l y$ ve $v=x r y$ olmasidir.

Bir $u \in X^{*}$ kelimesi için $u \rightarrow_{R} v$ olacak şekilde bir $v \in X^{*}$ kelimesi varsa bu $u$ kelimesine indirgenir kelime denir. Aksi durumda ise bu $u$ kelimesine indirgenemez kelime denir.

$u, v \in X^{*}$ için, eğer $u \rightarrow{ }_{R}^{*} v$ bağıntısı varsa ve $v$ kelimesi indirgenemez ise, bu $v$ kelimesine $u$ kelimesinin normal formu denir.

Tanım 2.1. (Book ve Otto, 1993) $R$ yeniden yazma sistemi olsun.

i) $R$ sistemi için kelimeler arasında $u_{1} \rightarrow u_{2} \rightarrow u_{3} \rightarrow \ldots$ şeklinde sonsuz bir zincir yoksa, bu yeniden yazma sistemine Noetherian ya da sona ermiş denir.

ii) $R$ sisteminden alınacak bütün $u, v, w \in X^{*}$ kelimeleri için, $u \rightarrow_{R}^{*} v$ ve $u \rightarrow_{R}^{*} w$ iken 
$v \rightarrow_{R}^{*} z \quad$ ve $w \rightarrow_{R}^{*} z$ olacak şekilde bir $z \in X^{*}$ kelimesi varsa, bu yeniden yazma sistemine elmas kuralını săglar (confluent) denir.

(iii) $R$ sistemi hem Noetherian hem de confluent özelliklerini sağlıyor ise bu yeniden yazma sistemine tam (complete ya da convergent) denir.

Eğer $R$ yeniden yazma sistemi tam ise, bu sistem içindeki her bir kelime tek bir normal forma sahip olduğundan, bu $R$ yeniden yazma sistemi çözülebilir kelime problemine sahiptir.

\subsection{Grupların çapraz çarpımı}

Agore ve Militaru (2008), Agore ve Fratila (2010), çapraz çarpım yapısını gruplar üzerinde çalışmış ve bu çarpımın bir grup olduğunu ispatlamışlardır. Ayrıca bileşenleri devirli gruplar alarak bu grupların çapraz çarpımlarının sunuşlarını elde etmişlerdir.

$H$ ve $G$ iki grup olmak üzere, herhangi $g, g_{1}, g_{2,} g_{3} \in G \quad$ ve $\quad h \in H \quad$ için $\alpha: G \rightarrow A u t(H), g \mapsto g \triangleright_{\alpha} h$ ve

$f: G \times G \rightarrow H$ dönüşümleri

$g_{1} \triangleright_{\alpha}\left(g_{2} \triangleright_{\alpha} h\right)=f\left(g_{1}, g_{2}\right)\left(g_{1} g_{2} \triangleright_{\alpha} h\right) f\left(g_{1}, g_{2}\right)^{-1}$

$f\left(g_{1}, g_{2}\right) f\left(g_{1} g_{2}, g_{3}\right)=\left(g_{1} \triangleright_{\alpha} f\left(g_{2}, g_{3}\right)\right) f\left(g_{1}, g_{2} g_{3}\right)$

koşullarını sağlıyorsa, bu durumda $H$ ve $G$ gruplarının çapraz sistemi $(H, G, \alpha, f)$ biçimindeki bir sıralı dörtlüdür. Eğer $\forall g \in G \quad$ için $\quad f(1,1)=f(1, g)=f(g, 1)=1$ ise, $(H, G, \alpha, f)$ çapraz sistemi normal çapraz sistem olarak adlandırılır. $(H, G, \alpha, f)$ çapraz sistemi ile birlikte $H$ ve $G$ 'nin çapraz çarpımı $H \#_{\alpha}^{f} G$ ile gösterilip $\left(h_{1}, g_{1}\right)\left(h_{2}, g_{2}\right)=\left(h_{1} \cdot\left(g_{1} \triangleright_{\alpha} h_{2}\right) f\left(g_{1}, g_{2}\right), g_{1} g_{2}\right)$ çarpımı ile tanımlanan $H \times G$ kümesidir.

Tanım 2.2. (Agore ve Militaru, 2008) $(H, G, \alpha, f)$ normal çapraz sistemi verilmiş olsun. $\forall h \in H$ ve $g \in G$ için (1) ile verilen çarpım altında $H \#_{\alpha}^{f} G$, birimi $(1,1)$ ve ters $\operatorname{eleman} 1(h, g)^{-1}=\left(f\left(g^{-1}, g\right)^{-1} g^{-1} \triangleleft_{\alpha} h^{-1}, g^{-1}\right)$ olan bir grup oluşturur.

İki grubun çapraz çarpımı; $\alpha$ ve $f^{\prime}$ nin özel durumlarına göre direkt, yarı-direkt ya da twisted çarpıma indirgenmektedir.

$C_{n}=\left\langle a ; a^{n}=1\right\rangle \quad$ ve $C_{m}=\left\langle b ; b^{m}=1\right\rangle$ sonlu devirli grup sunuşları ve $C_{g}=\langle g ;\rangle$ sonsuz devirli bir grup sunuşu olsun.

Teorem 2.1. (Agore ve Fratila, 2010)

(i) $C_{n} \#_{\alpha}^{f} C_{m}$ tipindeki çapraz çarpımının sunuşu

$C_{n} \#_{\alpha}^{f} C_{m}=\left\langle a, b ; a^{n}=1, b^{m}=a^{i}, b^{-1} a b=a^{j}\right\rangle$

biçimindedir. Burada $0 \leq i, j \leq n-1$, $i .(j-1) \equiv 0(\bmod n)$ ve $j^{m} \equiv 1(\bmod n) \operatorname{dir}$.

(ii) $C_{n} \#_{\alpha}^{f} C_{g}$ tipindeki çapraz çarpımının sunuşu $C_{n} \#_{\alpha}^{f} C_{g}=\left\langle a, g ; a^{n}=1, g^{-1} a g=a^{t}\right\rangle$ biçimindedir. Burada $(t, n)=1$ ve $t \in \mathbb{Z}$ dir.

(iii) $C_{g} \#_{\alpha}^{f} C_{n}$ tipindeki çapraz çarpımının sunuşu $\quad C_{g} \#_{\alpha}^{f} C_{n}=\left\langle g, h ; g h=h g, h^{n}=g^{t}\right\rangle$ biçimindedir. Burada $t \in \mathbb{Z}$ dir.

(iv) $C_{g_{1}} \#_{\alpha}^{f} C_{g_{2}}$ tipindeki çapraz çarpımının sunuşu $\quad C_{g_{1}} \#_{\alpha}^{f} C_{g_{2}}=\left\langle g_{1}, g_{2} ; g_{1} g_{2} g_{1}=g_{2}\right\rangle$ biçimindedir. 


\section{Sonuçlar}

Bu bölümde ilk olarak, Teorem 2.1 (iv) ile verilen rankları 1 olan iki serbest grubun çapraz çarpımının monoid sunuşu için tam yeniden yazma sistemi bulunacaktır. Daha sonra ise, rankları 2 olan iki serbest abelyan grubun çapraz çarpımının sunuşu elde edilecektir.

Teorem 2.1 (iv) ile verilen rankları 1 olan iki serbest grubun çapraz çarpımının $\left\langle\begin{array}{rl}g_{1}, g_{1}^{-1} g_{2}, g_{2}^{-1} & ; g_{1} g_{2} g_{1}=g_{2}, \\ & g_{1} g_{1}^{-1}=g_{1}^{-1} g_{1}=g_{2} g_{2}^{-1}=g_{2}^{-1} g_{2}=1\end{array}\right\rangle$

biçiminde monoid sunuşunu ele alalım. $\mathrm{Bu}$ sunuşun tam yeniden yazma sistemi için aşağıdaki sonuç elde edilmiştir. Bunun için kelimeler arasında uzunluk-harfsel siralama ve üreteçler arasında $g_{1}>g_{1}^{-1}>g_{2}>g_{2}^{-1}$ sıralamasını dikkate alalım. Ayrıca, (i) ve ( $j$ ) yeniden yazma kurallarındaki bağıntıları göstermek üzere, bu bağıntıların kesişerek elde edilen çakışan kelimeleri $(i) \cap(j)$ notasyonu ile gösterilmektedir.

Teorem 3.1. Rankları 1 olan iki serbest grubun çapraz çarpımının monoid sunuşunun tam yeniden yazma sistemi aşağıdaki kurallardan oluşmaktadır.
(1) $g_{1} g_{2} \rightarrow g_{2} g_{1}^{-1}$,
(2) $g_{1}^{-1} g_{2} \rightarrow g_{2} g_{1}$
(3) $g_{1}^{-1} g_{2}^{-1} \rightarrow g_{2}^{-1} g_{1}$,
(4) $g_{1} g_{2}^{-1} \rightarrow g_{2}^{-1} g_{1}^{-1}$
(5) $g_{1} g_{1}^{-1} \rightarrow 1$,
(6) $g_{1}^{-1} g_{1} \rightarrow 1$,
(7) $g_{2} g_{2}^{-1} \rightarrow 1$,
(8) $g_{2}^{-1} g_{2} \rightarrow 1$.

İspat. Yeniden yazma kurallarındaki üreteçler arasında $\quad g_{1}>g_{1}^{-1}>g_{2}>g_{2}^{-1}$ biçiminde bir harfsel sıralama olduğundan, kelimelerin indirgendiği kelimeler sonlu sayıda adımla elde edilir. Böylece, sistemin tam olması için ilk şart olan Noetherian özelliği sağlanmış olur. Confluent (elmas kuralı) özelliğinin sağlandığını göstermek için ise, yeniden yazma kurallarının çakışan bütün kelimeleri ve kritik çiftleri aşağıda verilmiştir.

(1) $\cap(7): g_{1} g_{2} g_{2}^{-1}, \quad\left(g_{2} g_{1}^{-1} g_{2}^{-1}, g_{1}\right)$,

(2) $\cap(7): g_{1}^{-1} g_{2} g_{2}^{-1}, \quad\left(g_{2} g_{1} g_{2}^{-1}, g^{-1}\right)$,

(3) $\cap(8): g_{1}^{-1} g_{2}^{-1} g_{2}, \quad\left(g_{2}^{-1} g_{1} g_{2}, g_{1}^{-1}\right)$,

(4) $\cap(8): g_{1} g_{2}^{-1} g_{2}, \quad\left(g_{2}^{-1} g_{1}^{-1} g_{2}, g_{1}\right)$,

(5) $\cap(2): g_{1} g_{1}^{-1} g_{2}, \quad\left(g_{2}, g_{1} g_{2} g_{1}\right)$,

(5) $\cap(3): g_{1} g_{1}^{-1} g_{2}^{-1}, \quad\left(g_{2}^{-1}, g_{1} g_{2}^{-1} g_{1}\right)$,

(6) $\cap(1): g_{1}^{-1} g_{1} g_{2}, \quad\left(g_{2}, g_{1}^{-1} g_{2} g_{1}^{-1}\right)$,

(6) $\cap(4): g_{1}^{-1} g_{1} g_{2}^{-1}, \quad\left(g_{2}^{-1}, g_{1}^{-1} g_{2}^{-1} g_{1}^{-1}\right)$,

$(5) \cap(6): g_{1} g_{1}^{-1} g_{1}, \quad\left(g_{1}, g_{1}\right)$,

(6) $\cap(5): g_{1}^{-1} g_{1} g_{1}^{-1}, \quad\left(g_{1}^{-1}, g_{1}^{-1}\right)$,

(7) $\cap(8): g_{2} g_{2}^{-1} g_{2}, \quad\left(g_{2}, g_{2}\right)$,

$(8) \cap(7): g_{2}^{-1} g_{2} g_{2}^{-1}, \quad\left(g_{2}^{-1}, g_{2}^{-1}\right)$.

Yukarıdaki bütün kritik çiftler, çakışan kelimeye bağlı olarak sonlu adımda kendi aralarında aynı kelimeye indirgendiğinden çözülebilirdir. Örneğin,

$(2) \cap(7): g_{1}^{-1} g_{2} g_{2}^{-1}, \quad\left(g_{2} g_{1} g_{2}^{-1}, g_{1}^{-1}\right)$,

$$
g_{1}^{-1} g_{2} g_{2}^{-1} \rightarrow\left\{\begin{array}{l}
g_{1}^{-1} \\
g_{2} g_{1} g_{2}^{-1} \rightarrow g_{2} g_{2}^{-1} g_{1}^{-1} \rightarrow g_{1}^{-1}
\end{array}\right.
$$

O halde yukarıda verilen yeniden yazöa sistemi Noetherian ve confluent olduğundan tamdir.

Sonuç 3.2. Rankları 1 olan iki serbest grubun çapraz çarpımının monoid sunuşu için elemanlarının normal formu $g_{2}{ }^{\varepsilon_{1}} g_{1}{ }^{\varepsilon_{2}}\left(\varepsilon_{1}, \varepsilon_{2} \in \mathbb{Z}\right)$ şeklindedir.

Teorem 3.1 ve Sonuç 3.2 ile verilen normal form yapısı kullanılarak aşağıdaki sonuç elde edilmiştir. 
Sonuç 3.3. Rankları 1 olan iki serbest grubun çapraz çarpımının monoid sunuşu için kelime problemi çözülebilirdir.

Agore ve Fratila (2010) sonlu/sonsuz devirli grupların çapraz çarpımının sunuşlarını elde etmişlerdir. Literatürde başka tip grupların çapraz çarpımının sunuşlarının elde edilmesi ile ilgili çalışmalar bulunmadığından bu yöndeki çalışmalar bu alanda önem kazanmaktadır. Dolayısıyla aşağıdaki sonuç rankları 2 olan serbest abelyan grupların çapraz çarpımının sunuşunu vermektedir. Bunun için $X=\left\langle x_{1}, x_{2} ; x_{1} x_{2}=x_{2} x_{1}\right\rangle \quad$ ve $Y=\left\langle y_{1}, y_{2} ; y_{1} y_{2}=y_{2} y_{1}\right\rangle \quad$ serbest abelyan gruplarını ele alalım. Ayrıca $\alpha: Y \rightarrow \operatorname{Aut}(X), y \mapsto \alpha_{y}(x)$ ve $f: Y \times Y \rightarrow X$ dönüşümler olsun.

Teorem 3.4. $X \#_{\alpha}^{f} Y \quad$ çapraz çarpımının sunuşu

$$
\left\langle\begin{array}{c}
x_{1}, x_{2}, y_{1}, y_{2} ; x_{1} x_{2}=x_{2} x_{1}, y_{1} y_{2} y_{1}^{-1} y_{2}^{-1}=W_{X}, \\
y_{i} x_{j}^{\varepsilon}=\alpha_{y_{i}}\left(x_{j}^{\varepsilon}\right) y_{i}, \\
y_{i}^{-1} x_{j}^{\varepsilon}=\left(W_{y_{i}^{-1}, y_{i}}^{-1} \alpha_{y_{i}^{-1}}\left(x_{j}^{\varepsilon}\right) W_{y_{i}^{-1}, y_{i}}\right) y_{i}^{-1}
\end{array}\right\rangle
$$

biçimindedir. Burada $W_{X}$ kelimesi $X$ grubunun üreteçleri ile elde edilen bir kelime, $W_{y_{i}^{-1}, y_{i}}=f\left(y_{i}^{-1}, y_{i}\right) \quad(1 \leq i, j \leq 2) \quad$ ve $\varepsilon= \pm 1$ dir.

İspat. $Z=\left\{x_{1}, x_{2}, y_{1}, y_{2}\right\}$ ve $\theta: Z^{*} \rightarrow X \#_{\alpha}^{f} Y$, $\theta\left(x_{i}\right)=\left(x_{i}, 1\right) \quad$ ve $\quad \theta\left(y_{j}\right)=\left(1, y_{j}\right)$ $(1 \leq i, j \leq 2) \quad$ biçiminde tanımlı bir homomorfizma olsun. $\mathrm{Bu} \theta$ homomorfizmasının örten olduğu kolaylıkla görülebilmektedir.
Şimdi $X \#_{\alpha}^{f} Y$ çapraz çarpımının (2) sunuşu ile verilen bağıntıları sağladığını sırasıyla gösterelim.

$$
\begin{aligned}
\left(x_{1}, 1\right)\left(x_{2}, 1\right) & =\left(x_{1} \alpha_{1}\left(x_{2}\right) f(1,1), 1\right) \\
& =\left(x_{1} x_{2}, 1\right)=\left(x_{2} x_{1}, 1\right)=\left(x_{2}, 1\right)\left(x_{1}, 1\right)
\end{aligned}
$$

dir. Ayrica

$\left(1, y_{1}\right)\left(1, y_{2}\right)\left(1, y_{1}\right)^{-1}\left(1, y_{2}\right)^{-1}$

$=\left(f\left(y_{1}, y_{2}\right), y_{1} y_{2}\right)\left(f\left(y_{1}^{-1}, y_{1}\right)^{-1}, y_{1}^{-1}\right)\left(f\left(y_{2}^{-1}, y_{2}\right)^{-1}, y_{2}^{-1}\right)$

$=\left(f\left(y_{1}, y_{2}\right), y_{1} y_{2}\right)(\underbrace{f\left(y_{1}^{-1}, y_{1}\right)^{-1} \alpha_{y-1}\left(f\left(y_{2}^{-1}, y_{2}\right)^{-1}\right) f\left(y_{1}^{-1}, y_{2}^{-1}\right)}_{F}, y_{1}^{-1} y_{2}^{-1})$

$=\left(f\left(y_{1}, y_{2}\right) \alpha_{y_{1} y_{2}}(F) f\left(y_{1} y_{2}, y_{1}^{-1} y_{2}^{-1}\right), y_{1} y_{2} y_{1}^{-1} y_{2}^{-1}\right)$

$=\left(W_{X}, 1\right)$

olup (2) sunuşundaki ilk iki bağıntı sağlanmaktadır. Şimdi

$$
y_{i} x_{j}^{\varepsilon}=\alpha_{y_{i}}\left(x_{j}^{\varepsilon}\right) y_{i} \quad(1 \leq i, j \leq 2)
$$

bağıntısının sağlandığını gösterelim. Bunun için,

$$
\begin{aligned}
\left(1, y_{i}\right)\left(x_{j}, 1\right)^{\varepsilon} & =\left(1, y_{i}\right)\left(x_{j}^{\varepsilon}, 1\right) \\
& =(\alpha_{y_{i}}\left(x_{j}^{\varepsilon}\right) \underbrace{f\left(y_{i}, 1\right)}_{1}, y_{i}) \\
& =\left(\alpha_{y_{i}}\left(x_{j}^{\varepsilon}\right), y_{i}\right) \\
& =\left(\alpha_{y_{i}}\left(x_{j}^{\varepsilon}\right), 1\right)\left(1, y_{i}\right)
\end{aligned}
$$

dir. (2) sunuşundaki son bağıntı için,

$$
\begin{aligned}
\left(1, y_{i}\right)^{-1}\left(x_{j}, 1\right)^{\varepsilon} & =\left(f\left(y_{i}^{-1}, y_{i}\right)^{-1} \alpha_{y_{i}^{-1}}(1), y_{i}^{-1}\right)\left(x_{j}^{\varepsilon}, 1\right) \\
& =\left(f\left(y_{i}^{-1}, y_{i}\right)^{-1}, y_{i}^{-1}\right)\left(x_{j}^{\varepsilon}, 1\right) \\
& =(f\left(y_{i}^{-1}, y_{i}\right)^{-1} \alpha_{y_{i}^{-1}}\left(x_{j}^{\varepsilon}\right) \underbrace{f\left(y_{i}^{-1}, 1\right)}_{1}, y_{i}^{-1}) \\
& =\left(f\left(y_{i}^{-1}, y_{i}\right)^{-1} \alpha_{y_{i}^{-1}}\left(x_{j}^{\varepsilon}\right), y_{i}^{-1}\right)
\end{aligned}
$$

$=\left(f\left(y_{i}^{-1}, y_{i}\right)^{-1} \alpha_{y_{i}^{-1}}\left(x_{j}^{\varepsilon}\right) f\left(y_{i}^{-1}, y_{i}\right), 1\right)\left(f\left(y_{i}^{-1}, y_{i}\right)^{-1}, y_{i}^{-1}\right)$

$=\left(f\left(y_{i}^{-1}, y_{i}\right)^{-1} \alpha_{y_{i}^{-1}}\left(x_{j}^{\varepsilon}\right) f\left(y_{i}^{-1}, y_{i}\right), 1\right)\left(1, y_{i}\right)^{-1}$

$=\left(W_{y_{i}^{-1}, y_{i}}^{-1} \alpha_{y_{i}^{-1}}\left(x_{j}^{\varepsilon}\right) W_{y_{i}^{-1}, y_{i}}\right) y_{i}^{-1}$ 
elde edilir. Böylece bu $\theta$ homomorfizması, (2) sunuşu ile tanımlı bir $G$ grubundan $X \#_{\alpha}^{f} Y$ çapraz çarpım grubu üzerine bir $\bar{\theta}$ epimorfizması vermektedir. Son olarak bu $\bar{\theta}$ homomorfizmasının birebir olduğunu gösterelim. Bunun için $w \in Z^{*}$ boştan farklı herhangi bir kelime olsun. (2) sunuşundaki bağıntılar ile $w=w_{x} w_{y} \in G$ olacak şekilde $w_{x} \in\left\{x_{1}, x_{2}, x_{1}^{-1}, x_{2}^{-1}\right\}^{*}$ ve $w_{y} \in\left\{y_{1}, y_{2}, y_{1}^{-1}, y_{2}^{-1}\right\}^{*}$

kelimeleri vardır. Böylece bu $w \in Z^{*}$ kelimesi

$$
\begin{aligned}
\bar{\theta}(w) & =\theta(w)=\theta\left(w_{x}, w_{y}\right)=\theta\left(w_{x}\right) \theta\left(w_{y}\right) \\
& =\left(w_{x}, 1\right)\left(w_{x^{\prime}}, w_{y}\right) \\
& =\left(w_{x} w_{x^{\prime}}, w_{y}\right)
\end{aligned}
$$

için,

$\left(w_{x^{\prime}} \in\left\{x_{1}, x_{2}, x_{1}^{-1}, x_{2}^{-1}\right\}^{*}\right)$ elde edilir. Şimdi $w^{\prime}=w_{x}^{\prime} w_{y}^{\prime}, w^{\prime \prime}=w_{x}^{\prime \prime} w_{y}^{\prime \prime} \in Z^{*} \quad$ kelimelerini alalım ve $\theta\left(w^{\prime}\right)=\theta\left(w^{\prime \prime}\right)$ olsun. Buradan (3) ile $w_{x}^{\prime} w_{x^{\prime}}^{\prime}=w_{x}^{\prime \prime} w_{x^{\prime}}^{\prime \prime} \in X \quad$ ve $w_{y}^{\prime}=w_{y}^{\prime \prime} \in Y$ elde edilir. Burada $w_{y}^{\prime}=w_{y}^{\prime \prime}$ olduğu için, $w_{x^{\prime}}^{\prime}=w_{x^{\prime \prime}}^{\prime \prime}$ dir. Böylece $w_{x}^{\prime}=w_{x}^{\prime \prime} \in X$ eşitliği elde edilir. $\mathrm{Bu}$ durumda $w_{x}^{\prime}=w_{x}^{\prime \prime}$ ve $w_{y}^{\prime}=w_{y}^{\prime \prime}$ eşitlikleri $G$ grubunda sağlanmaktadır. $\mathrm{O}$ halde, $w^{\prime}=w^{\prime \prime}$ ve $\bar{\theta}$ homomorfizmas1 birebirdir. Sonuç olarak (2) sunuşu ile verilen $G$ grubu $X$ \# ${ }_{\alpha}^{f} G$ çapraz çarpım grubuna izomorftur.

Teorem 3.4 ile verilen $X \#_{\alpha}^{f} Y \quad$ çapraz çarpımının sunuşunun tam yeniden yazma sistemi bağıntı kümesinde verilen $W_{y_{i}^{-1}, y_{i}}$ ve $W_{X}$ kelimelerinin uzunluklarına bağlı olduğu için, bu yöndeki bir çalışmanın bu kelimelerin özel durumları düşünülerek ilerideki bir zamanda yapılması planlanmaktadır. Ayrıca çapraz çarpımı oluşturan grupların keyfi gruplar alınarak bu grupların çapraz çarpımının sunuşunun elde edilmesi açık bir problemdir.

\section{Açıklamalar}

$\mathrm{Bu}$ çalışma 32. Ulusal Matematik Sempozyumunda (UMS 2019) sunulmuştur.

\section{Kaynaklar}

Abuhlail, J. Y. 2005. "Duality Theorems for Crossed Products over Rings", Journal of Alg., 288 (1), 212-240.

Agore, A. L. ve Militaru, G. 2008. "Crossed Product of Groups, Applications", Arab. J. Sci. Eng., 33, 1-17.

Agore, A. L. ve Fratila, D. 2010. "Crossed Product of Cyclic Groups", Czechoslov. Math. J., 60(135), 889-901.

Book, R. V. 1987. "Thue Systems as Rewriting Systems", J. Symbolic Computation, 3(1-2), 39-68.

Book, R. V. ve Otto, F. (1993). "String Rewriting Systems", Springer-Verlag, New York.

Carbani,G., Guccione, J. A. ve Guccione, J. J. 2010. "Cyclic Homology of Hopf Crossed Products", Adv. in Math., 223(3), 840-872.

Çetinalp, E. K. 2016 "Bazı Grup ve Monoid Yapıları için Karar Verme Problemleri ve Büyüme Serileri”, Yüksek Lisans Tezi, Karamanoğlu Mehmetbey Üniversitesi Fen Bilimleri Enstitüsü, Karaman.

Çetinalp, E. K., Karpuz, E. G. , Ateş, F. ve Çevik, A. S. 2016. "Two-Sided Crossed Products of Groups", Filomat, 30 (4), 10051012.

Çetinalp, E. K. ve Karpuz, E. G. 2018. "Iterated Crossed Product of Cyclic Groups", Bulletin of the Iranian Math. Society, 44(6), 1493-1508. 
Dehn, M. 1911. "Uber unendliche diskontinuerliche Gruppen", Math. Ann., 69, 116-144.

Grundling, H. ve Neeb, K. H. 2014. "Crossed Products of $C^{*}$-Algebras for Singular Actions", Journal of Func. Analysis, 266 (8), 5199-5269.

Emin, A., Ateş, F., İkikardeş, S. ve Cangül, İ. N. 2013. "A New Monoid Construction under Crossed Products", Journal of Inequalities and Applications, 244.

Karpuz, E. G. ve Çetinalp, E. K. 2016. "Growth Series of Crossed and Two-Sided Crossed Products of Cyclic Groups", Mathematica Slovaca, 68(3), 537-548.

Miller, F. C. (1992). "Decision Problems For Groups-Survey and Reflections in Algorithms and Classification in Combinatorial Group Theory", MSRI Publications, Springer-Verlag, 23, 1-59.

Rudkovskii, M. A. 1997. "Twisted Product of Lie Groups", Siberian Math. Journal, 38, 1120- 1129.

Sims, C. C. (1994). "Computation with Finitely Presented Group", Cambridge University Press. 